\title{
Software and Algorithm of Drawing Surfaces
}

\author{
Kazushi AHARA and Yuhei YAMAZAKI \\ Department of Mathematics, Meiji University, Kawasaki 214-8571, Japan
}

Received July 1, 2002; final version accepted January 31, 2003

\begin{abstract}
In this paper, we shall consider a new kind of drawing software only for surfaces. We introduce pipe structure in pictures of surfaces. And the software Elephant allows us to draw surfaces easily and intuitively. This is a free software and we can get it at http://www.geocities.co.jp/CollegeLife-Labo/9021/.
\end{abstract}

KEYWORDS: surface, hyperbolic plane, Riemann surface, drawing software

\section{Introduction}

When we make figures of surfaces for a book or a paper of our own, we have some ways to do that. For example, we draw figures by hand and ask the publisher to make fair copies of them. Recently we often draw figures using a drawing software (for example, Adobe Illustrator [1].) and send the eps file to the publisher. But many of us feel that it is hard to draw figures which are just same as the ones we have in our imagination. Because if we asked a publisher to draw figures, it is hard to get across his images. If we uses a drawing software, it may take much time to do efficiently (and sometimes it takes more time to draw figures than to write texts.) It is because any drawing software is developed for general users not for only mathematicians.

Figures of surfaces consist of segments, ellipses, and Bezier curves which are sometimes thick, sometimes dotted, sometimes tangential together. Apparently drawing figures are a kind of delicate works and it takes much time to draw good figures.

It is very useful if we have a drawing software which allows us to draw pictures of surfaces easily and intuitively. The authors develop a new kind software Elephant under this concept. In this paper we shall introduce Elephant and argue some algorithms in it.

We shall explain the main idea of Elephant. We consider three pictures of surfaces as follows (Figs. 1.1a, 1.1b, 1.1c). Figure 1.1a is a torus and the other two are closed surfaces of genus two. Here we understand followings tacitly.

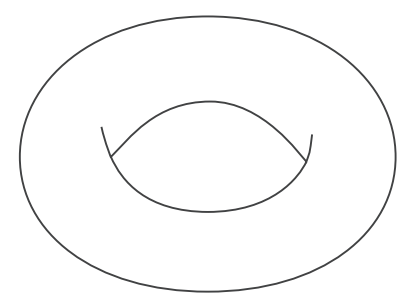

(a)

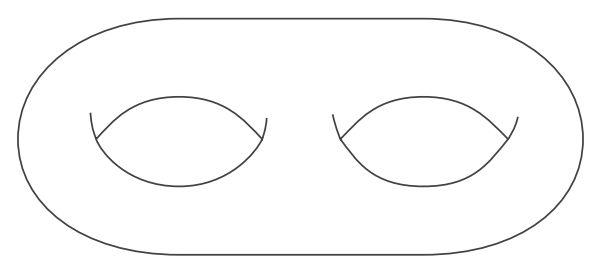

(b)

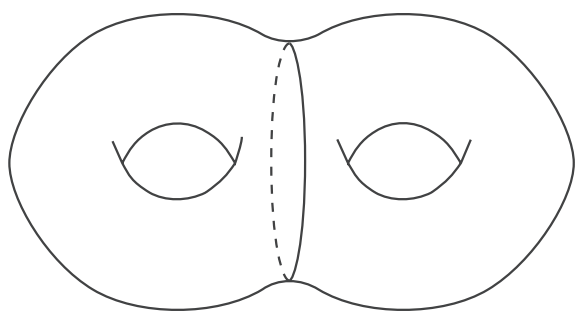

(c)

Fig. 1.1.

(1) A ringed pipe is drawn as a picture of a torus.

(2) When we draw a picture of a ringed pipe, we see it from a bird's-eye viewpoint. That is, there are two (external and internal) outlines, the external one is a large oval, and the internal one is a small oval with two angles and two small segments at the side. (We call this internal outline eyelid because if you turn this upside down, the picture looks like an eyelid.)

(3) The picture of the genus 2 surface has three outlines (one is external and the other two are internal), the external one is a large oval (see Fig. 1.1b) (or sometimes the external outline is drawn as in Fig. 1.1c) and the two internal outlines are eyelids.

We consider how we construct a data structure for drawing these pictures. We often draw a picture of a surface as a union of curved tubes and joints like water pipes. So it is very convenient that if we keep data of the center curves of such tubes. We call the set of such center curves a smooth bone. For example, for a torus figure, the bone is an oval. 
For a picture of a surface of genus 2 like Fig. 1.1b, the bone is a $\pi / 2$-rotated $\theta$ figure. For a picture of a surface of genus 2 like Fig. 1.1c, the bone is an infinity figure. See Fig. 1.2.

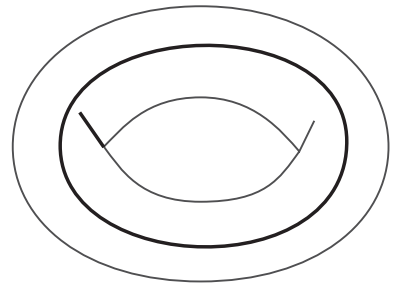

(a)

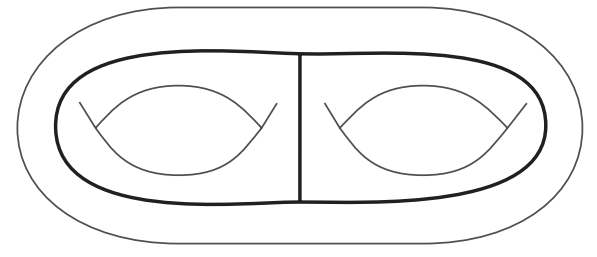

(b)

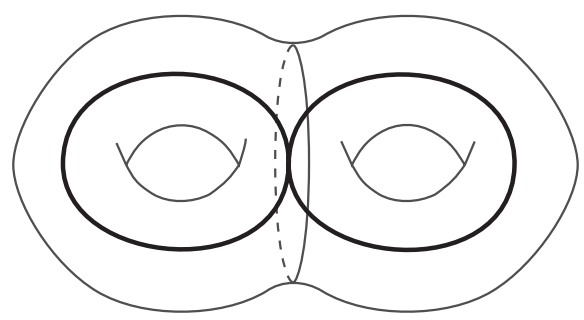

(c)

Fig. 1.2.

According to this first idea, Elephant may have the following feature. It allows users to draw a smooth bone and input the radius of the pipe, and afterward automatically we get a picture of a surface. The smooth bone is an embedded planer graph with curved edges, and the desirable outlines are 'offset' curves of the bone.

But when we input a smooth bone, we have to draw Bezier curves on the screen. This might not be so easy for novice users. We need an extended idea. That is, let a bone be a planer graph with only straight edges and the software draw the outlines according to circumstances. See Fig. 1.3.

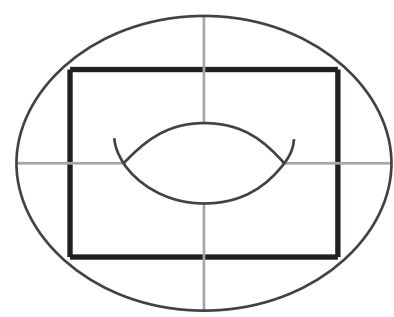

(a)

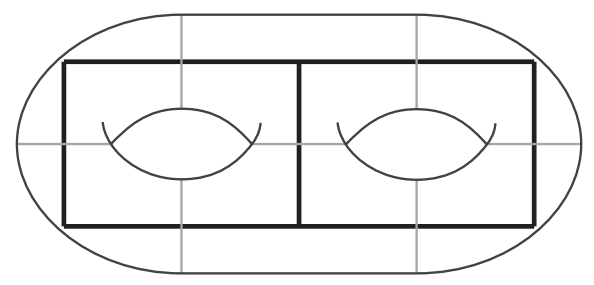

(b)

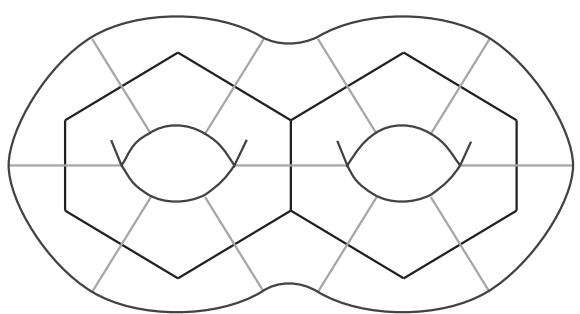

(c)

Fig. 1.3.

Clearly it is much easier to draw a straight line graph. So we use this second idea. See the definition of a bone in Sect. 2.

Furthermore Elephant allows us to export to the PostScript [2] format. So we easily reedit the figure by a drawing software and we easily insert these as figures in $\mathrm{T}_{\mathrm{E}} \mathrm{X}[3]$ documentation.

We suppose that main users of Elephant are topologists. The authors asked Prof. Kiyoshi Ohba (Ochanomizu women's university) to be a monitor of Elephant for three months. The authors would like to thank him very much. He wrote many reports to the authors and Elephant was enriched very much.

The author thanks Prof. Atsushi Sato and Prof. Masashi Katsurada for their advice and encouragement.

\section{Surveys of Pictures of Surfaces}

We often see figures of surfaces in papers and books. In most cases, a figure has a certain structure. For example, the surface may be a hyperbolic surface or it may be a complex line or it may be a minimal surface. The surface may have a normal crossing point or a cusp or an open boundary. Sometimes we want to emphasize that the surface is not simply connected or that it has a Morse function on it. Figures must be drawn such that it inspires us with such structures. In this section we shall survey pictures of surfaces.

First we argue pipe structure of a picture of a surface. We usually draw a picture of a surface as a jointed pipe. We say that such picture has pipe structure. This structure must have two components, a bone and a radius parameter. That is, there are center lines of pipes, and the union of these center lines constructs a graph. We call this graph $a$ bone. And each pipe has a radius. We call this a radius parameter.

\subsection{Pipe structure}

We consider pipe structure in a figure of a surface in the following steps. When we draw a picture of a surface we often draw it as follows. 
(1) There is the front and the back.

(2) There is lines between the front and the back. Usually there are upper (or right) lines and lower (or left) lines.

(3) We draw boundaries and sections as ovals.

See Fig. 2.1.

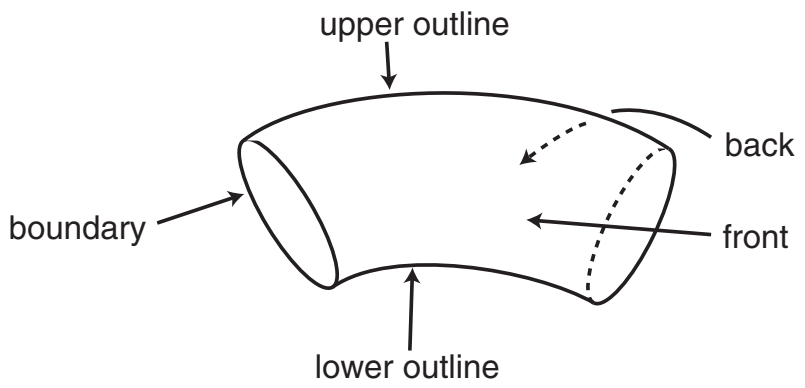

Fig. 2.1.

We call such structure of figures pipe structure. It is because we draw a picture of a surface like a pipe. A pipe is determined by the following two parts. One is the center line of the pipe (we call this a smooth bone) and the other is the radius of the pipe.

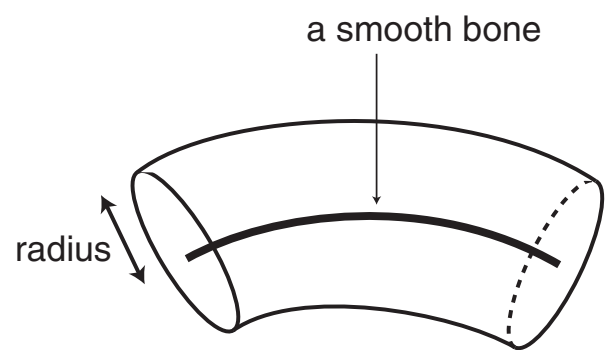

Fig. 2.2.

If a smooth bone and a radius parameter are given, we draw the picture of the pipe as follows.

(D1) The upper line and the lower line are offset curves of the smooth bone.

(D2) Two ends of the pipe are ovals such that the long axis of each oval is perpendicular with the smooth bone and the length of the length of the long axis is twice as the radius.

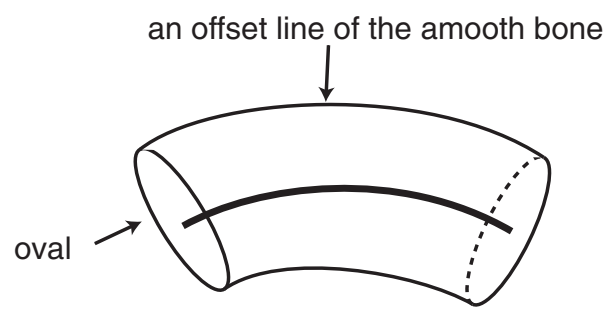

Fig. 2.3.

Here we have to mention what is a desirable user interface of Elephant. If a user draws a smooth bone and he/she inputs the radius of the pipe, he/she must draw a smooth curve on the drawing plane. Is it easy and intuitive? Demanding users to draw curves is against the first concept of this project. We have to redefine $a$ bone. A bone of $a$ single curved pipe is a union of two segments $A B$ and $B C$ such that:

(a) Two segments meet at a point $B$.

(b) The other ends $A, C$ of segments are the ends of the smooth bone, and the segments are tangential to the smooth bone at the end points.

When we get a bone in this mean and a radius, we draw a picture of the pipe as follows 


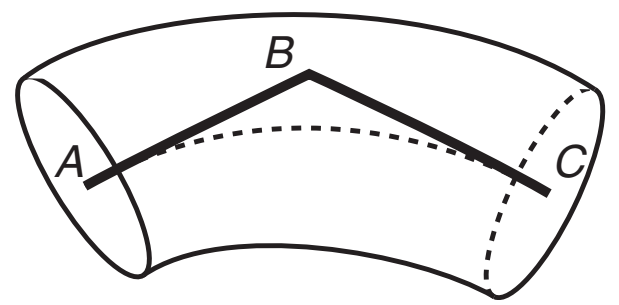

Fig. 2.4.

(D0) We determine four points of the ends of upper and lower outlines. That is, $D, E$ are the ends of upper outlines and $F, G$ are ends of lower outlines, such that $D F \perp A B$ and $A D=A F$ is the radius at $A$, and that $E G \perp B C$ and $C E=C G$ is the radius at $C$.

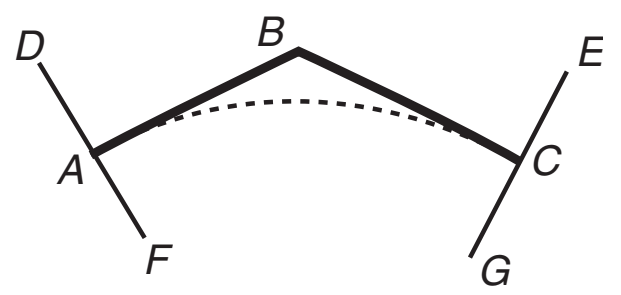

Fig. 2.5.

(D1') The upper outline is a curve such that it's perpendicular to $D F$ at $D$, and it is perpendicular to $E G$ at $E$. The lower outline is a curve such that it's perpendicular to $D F$ at $F$, and it is perpendicular to $E G$ at $G$.

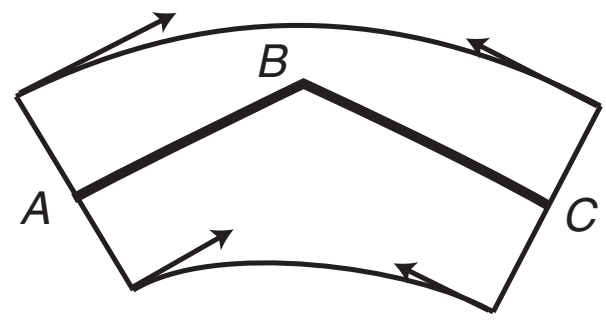

Fig. 2.6.

(D2) The two ends of the pipe are ovals with their long axes are $E G$ and $D F$ respectively.

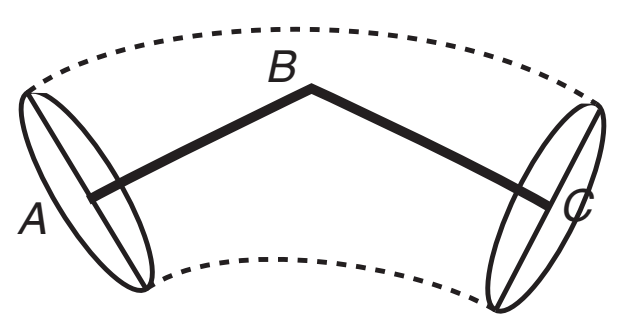

Fig. 2.7.

There are two remarks here. First we remark that we are allowed to vary the radius of the pipe for each ends of a bone. That is, we may consider the following as a picture of a surface with pipe structure.

Secondly, we remark that there is ambiguity to draw the upper outline and lower outline. There are two ways on this point. One is that computer draws a 'comfortable' outline automatically. The other is that a user inputs the curvature of the curve by hand. The first way (automatical way) is better for the concept of Elephant. See Sect. 4.4 for automatically determining of outline. 


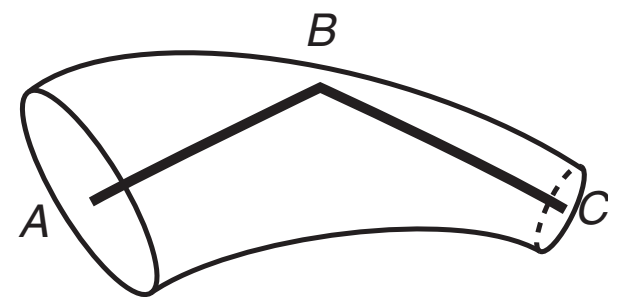

Fig. 2.8.

Next joint two bones to each other. The pipe is smooth totally, so we need the following condition.

Condition: If a bone $A B \cup B C$ and another bone $C D \cup D E$ are jointed together, then the meet point $C$ must be on the segment $B D$.

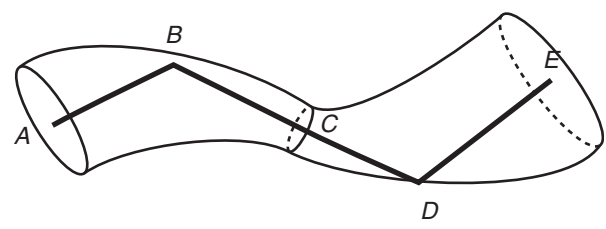

Fig. 2.9.

Under this condition, we may connect surfaces with pipe structure if the two radius parameters at the boundary coincide.

\subsection{Pants figure}

If we draw a picture of a surface of genus 2, the picture must contains a pants figure. Here a pants figure is as in Fig. 2.10. This is homotopic to a sphere with three holes.

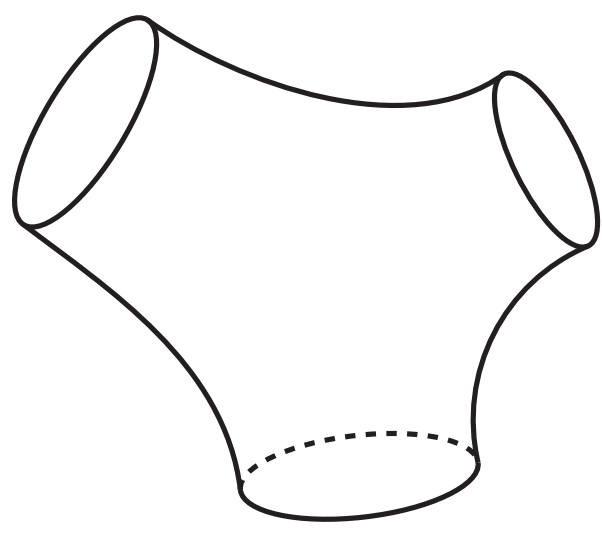

Fig. 2.10.

It is easy to extend pipe structure to a picture of pants. We provide an extended pipe structure (in the sequel we call it pipe structure again) as follows.

(a) A bone of a pants figure is an union of three straight segment which meet at one point.

(b) For another end of each segment, it has a radius parameter.

Remark: Mathematically we may consider a picture such that the three segments doesn't meet at one point. But we think that Fig. 2.11 is natural enough and we needn't consider such odd shapes. In the sequel we assume that the bone at pants figure is a tripod, a union of three segment meeting at one point.

We shall show the process to draw a picture of a pants figure from data of a bone and radius parameters. Let $A B \cup A C \cup A D$ be a bone. Let $r_{1}, r_{2}, r_{3}$ be radius parameters at $B, C, D$ respectively.

Let $E F$ be a segment such that $E F$ meets $A B$ at $B$, that $E F \perp A B$, and that $B E=B F=r_{1}$. Let $G H, I J$ be segments determined in the same way for $A C, A D$ respectively.

We draw a curve from $E$ to $J$ such that it is perpendicular to $E F$ and $I J$. In the same way we draw curves from $F$ to $G$ 


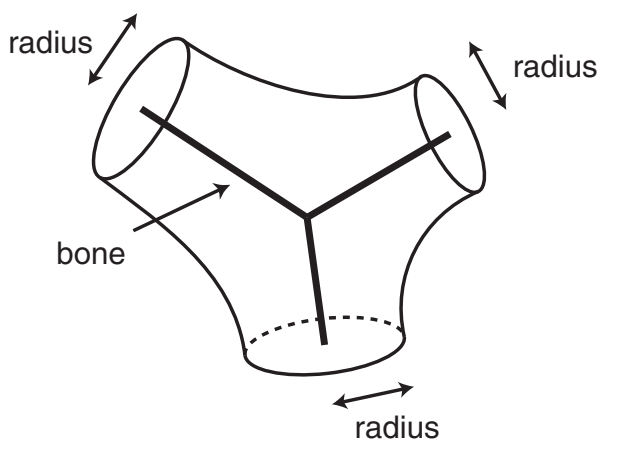

Fig. 2.11.

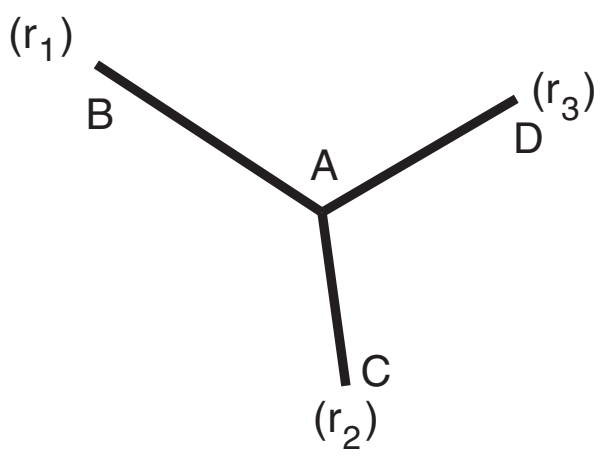

Fig. 2.12 .

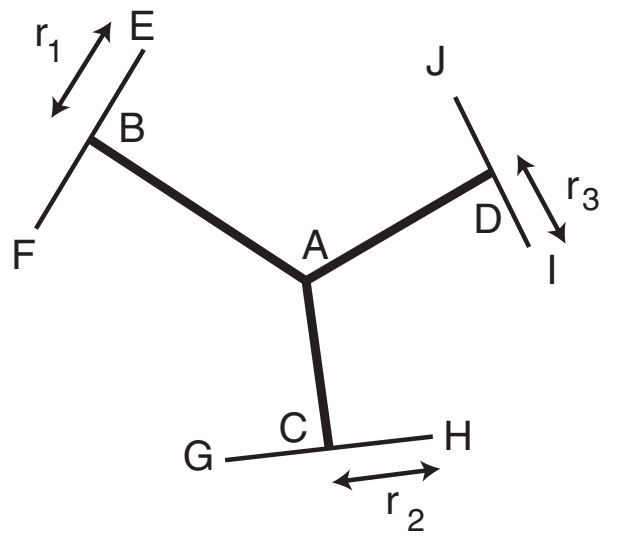

Fig. 2.13.

(resp. from $H$ to $I$ ).

At the boundary of pants figure, we draw three ovals such that long axes are $E F, G H, I J$ respectively. And we obtain a figure of a pants figure. In this figure we have some ambiguities. One is a curvature of the outlines. The other is a length of short axis of the boundary. In Elephant these parameters are automatically determined by the software.

Remark: To draw the outlines of pants, we need to mention the orientation of the three segments $A B, A C, A D$. (Remark that not the orientation of the triangle $B C D$ !) The following Fig. 2.16 is an erratum. We shall show an algorithm to avoid this figure in the Sect. 4.5.

\subsection{End of the pipe}

When we draw a picture of a surface with boundary, generally the following two cases may occur.

(a) A surface which we get by removing a disk from a surface (Fig. 2.17).

(b) A boundary of a cylinder (Fig. 2.18).

In the case (a), a picture of a boundary isn't compatible with pipe structure. But we always draw a circle or an oval as a boundary, so we apply Oval in Sect. 2.8.1 to this case. 


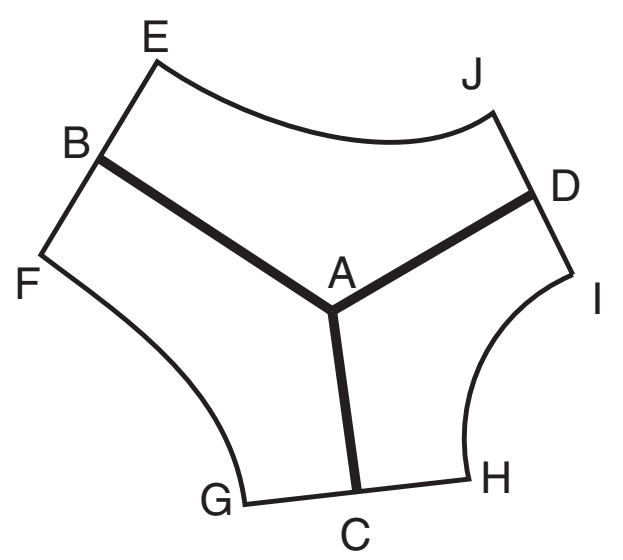

Fig. 2.14.

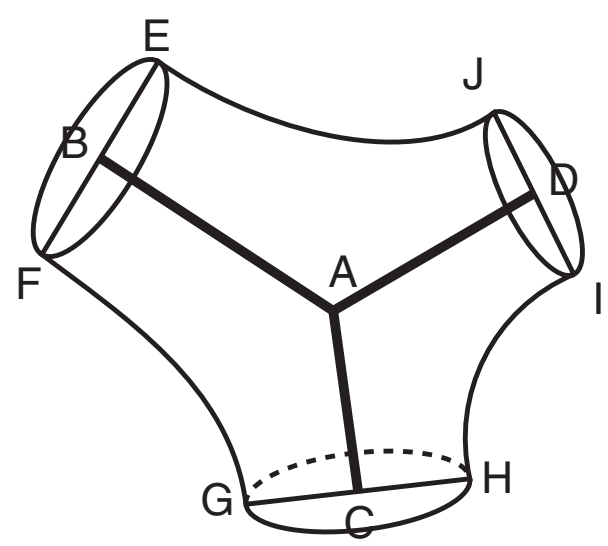

Fig. 2.15 .

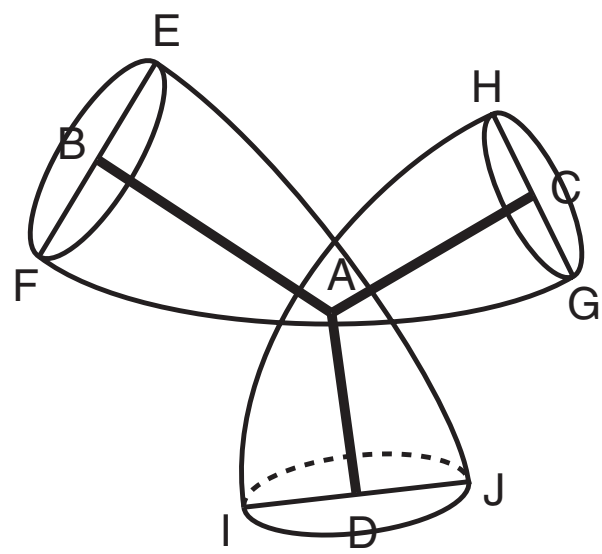

Fig. 2.16.

In the case (b), we can expand pipe structure to boundary. We define pipe structure at a boundary of the case (b) as follows.

(1) A bone consists of one straight segment.

(2) One of ends of the segment has a radius parameter.

(3) The other end of the segment has two data, boundary view and boundary shape. About these two data, see Sect. 2.6. 


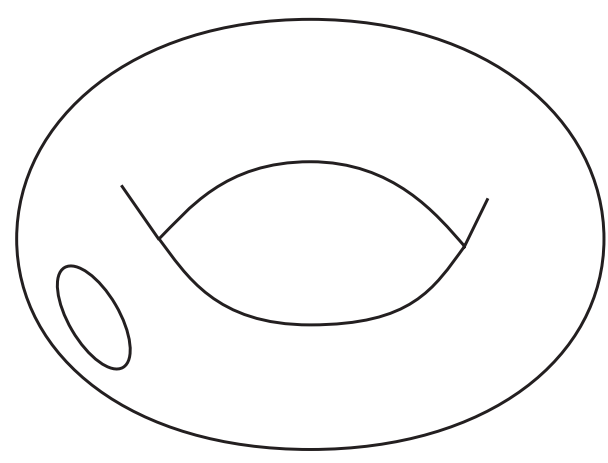

Fig. 2.17.

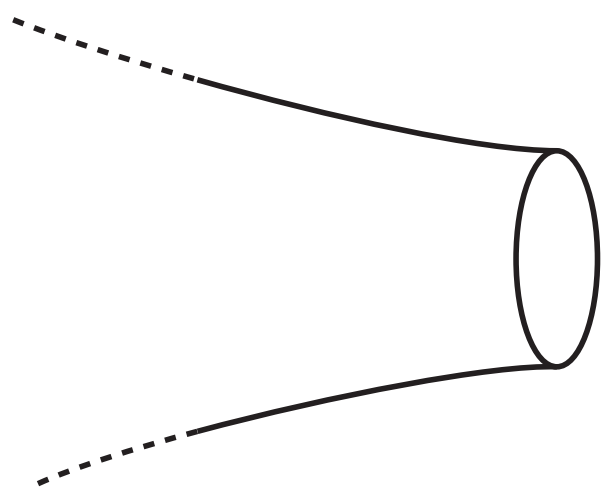

Fig. 2.18

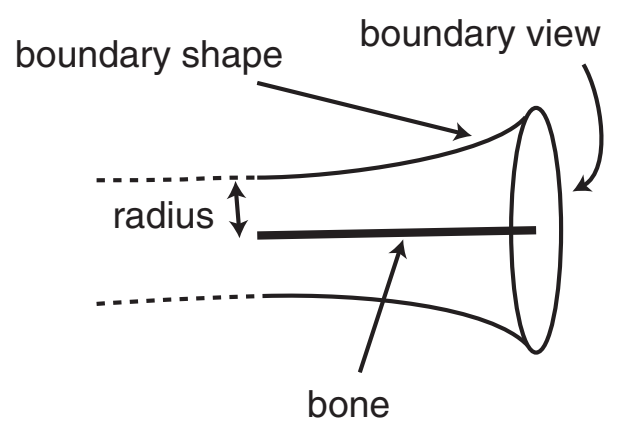

Fig. 2.19.

\subsection{Bone graph and gauge}

After all, we get a concept of bone structure as follows. We use a term radius parameter in the above section, but introducing a new concept gauge on a edge allows us to understand bone structure more clearly.

(1) A bone is a planer graph such that all edges are straight segments and that at most three edges meet at one vertex. (That is, the degree of each vertex is equal or less than 3.)

(2) For each edge, there are perpendicular segment, which is called gauge. An edge and the gauge of the edge meet at the midpoint of the gauge.

\subsection{Eyelid phenomenon}

When we draw a picture of a ringed pipe, we usually deform the internal outlines as follows.

In such figure the internal outline is not an oval, but it has two angles and short curved segments at both side. These short segments make us understand that the figure is a three dimensional. We call this local figure eyelid. Indeed, if we draw the same figure using raytracing method, we can see the similar line at both sides of the internal outlines.

Here we needn't the explicit formula for these small segments of eyelid. We feel that the eyelid outline is three dimensional not because the lines arises from the raytracing figure, but because the eyelid shape make us feel so. That is, eyelid is a kind of an optical illusion. 


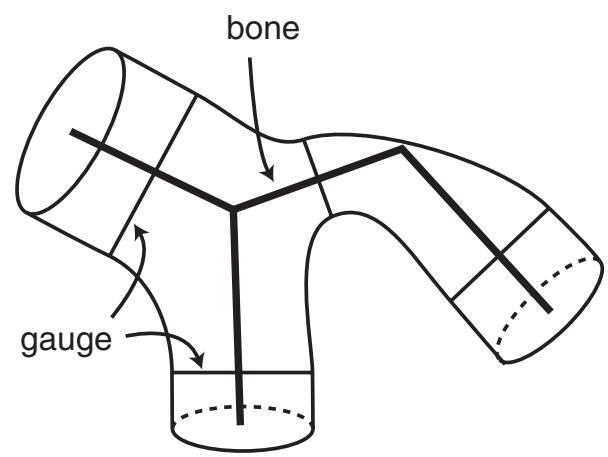

Fig. 2.20.

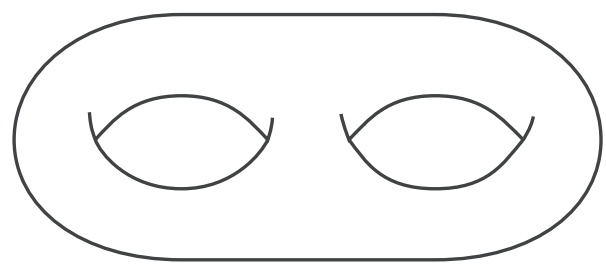

Fig. 2.21.

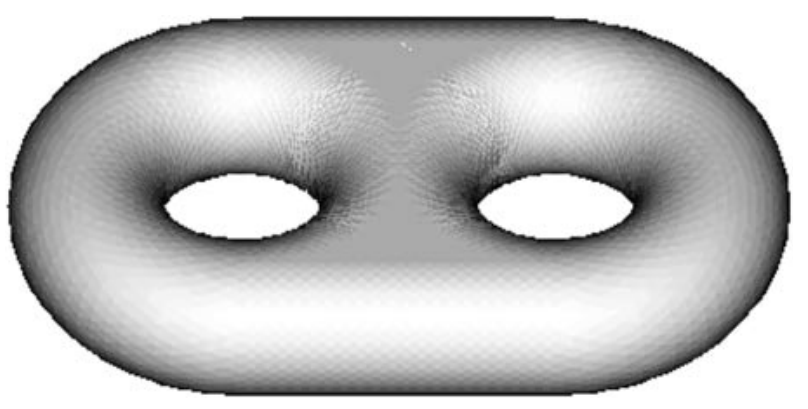

Fig. 2.22.

An eyelid figure appears mainly in the case that there is a horizontal gauge. Other than that, we use an eyelid figure when we want to emphasize that the surface has a positive genus like Fig. 2.24.

In the first case, this phenomenon occurs only if a gauge is horizontal (that is, the outline is vertical). So we make eyelid mode as a universal mode and we can set it on or off in the software. See Fig. 2.23.

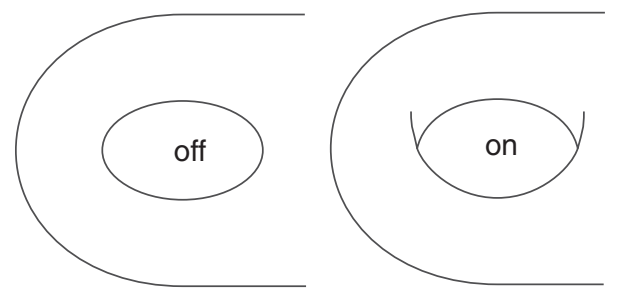

Fig. 2.23.

In the second case, eyelid figure is not compatible with pipe structure. So we make eyelid mode in oval element. If we draw an oval element along a slant line then the following figure may happen. It is clear that such eyelid figure is impossible in a raytracing way. But we dare not to exclude this case, because we sometimes need such figure.

\subsection{Boundary view and boundary shape}

As mentioned in the section of pipe structure, each degree 1 vertex has two parameters, boundary view parameter and boundary shape parameter. In this section we shall introduce these. 


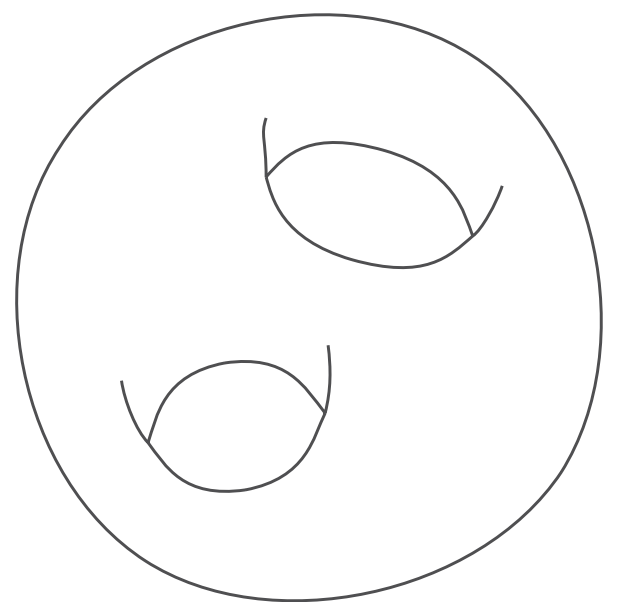

Fig. 2.24 .

Boundary shape parameter determines the shape of outlines near boundary. The followings are available now.

- Cylinder

Cylinder with a constant radius.

- Cusp

With a cusp singular.

- Cone

With a cone singular.

- Hemisphere

Capped by a hemisphere.

- Fadecone

Getting narrow at he boundary but not closed. We can set the rate of its narrowness from $0 \%$ to $100 \%$.

- Spleading 1

Getting wide at the boundary.

- Spleading2

Getting wider than Spleadingl.

- Hyperboric

Getting wide like a hyperbolic plane.

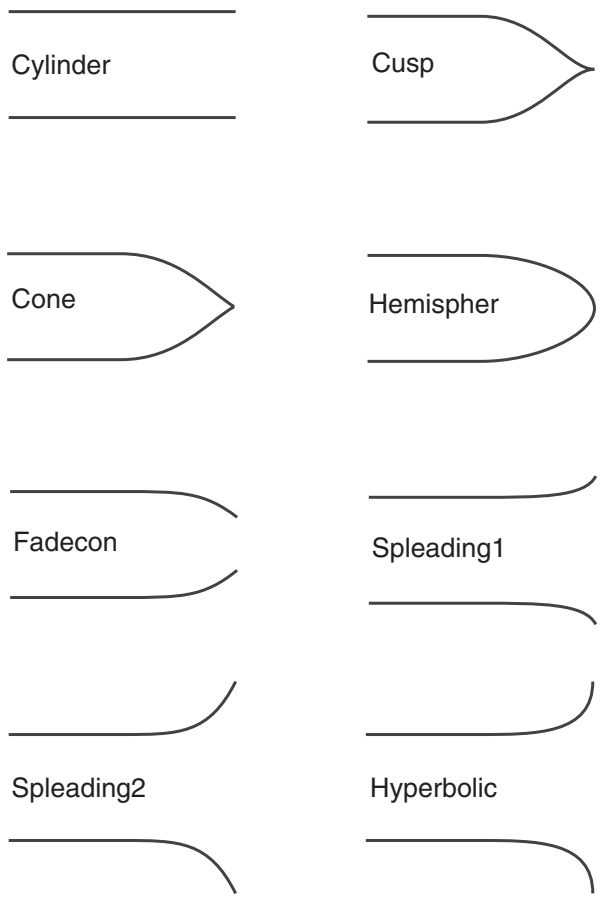

Fig. 2.25 . 
If we select Fadecone in Boundary shape, we may input how wide the boundary is. Default value is $50 \%$. If we input 0\% then Fadecone becomes Cone. If we input 100\% then Fadecone becomes Cylinder.

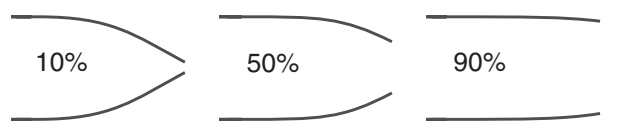

Fig. 2.26.

Boundary view parameter determines a shape of the section at the boundary. The followings are available now.

- None

No line at the boundary.

- Line

A straight segment.

- Ovall

A full oval of solid line.

- Oval2

A full oval of half solid (outside) and half dotted (inside).

- Half Oval

A half oval of solid line.

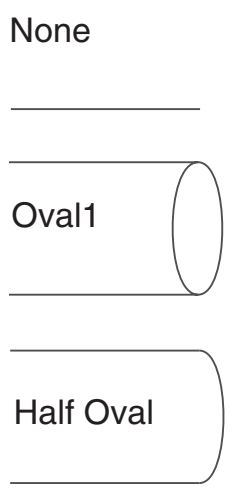

Line

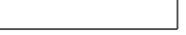

Oval2

Fig. 2.27.

Remark: We have ambiguity about a length of the short axis of the oval. If we draw pictures in raytracing method, the ratio between the short axis and the long axis may have various values. The authors find a natural ratio of between the long axis and the short axis. This ratio is used in Elephant.

Any of boundary view parameter can be compatible with boundary shapes except Cusp, Cone, Hemisphere. See the following examples.
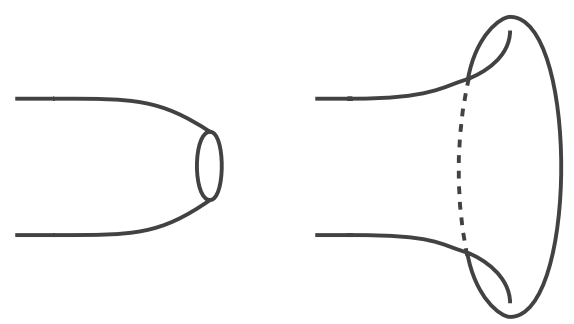

Fadecone+Oval1 Hyperbolic+Oval2

Fig. 2.28. 


\subsection{Pinching and section view}

When we draw a surface with singular point, it may happen to draw a pinching shape or tangential shape. So, we make some choices for pinching at the gauge of each edge.

- None

Usual pipe.

- Pinching

Pinching shape, that is two outlines is tangential at the center of the gauge.

- Normal cross

Two outlines intersect vertically at the center of the gauge.

- Sausage-tangential

At the center of the gauge two hemispheres meet tangentially.

- Bubble-tangential

At the center of the gauge two bubble-like ovals meet tangentially.

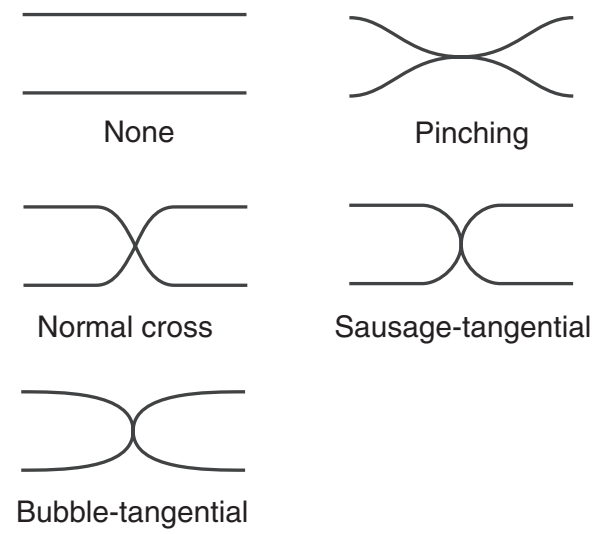

Fig. 2.29.

\subsubsection{Section view}

At the gauge of each edge, we may draw a section. This is a kind of an optical illusion and this section make us feel it three dimensional. The following choices are available, but Pinching shape parameter must be None.

- None

Nothing is drawn.

- Ovall

A full oval with half dotted and half solid.

- Oval2

Same as Ovall but the direction is reverse.

- Half Oval 1

A half oval.

- Half Oval 2

Same as Half Oval 1 but the direction is reverse.

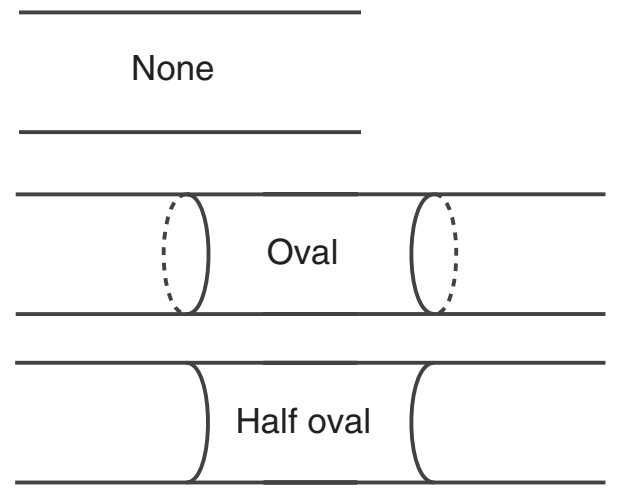

Fig. 2.30 . 


\subsection{Additional elements}

\subsubsection{Oval element and segment}

Oval element is an additional elements in the drawing plane. It has the similar structure as a bone with one edge. Its outline is always an oval, such that the edge is one axis and the gauge is another axis. If we set the length of the gauge is zero, Oval element becomes a segment. Oval element can exist independently from a bone, that is, the edge of Oval element may intersect a bone. We use Oval element in the following case.

(1) We may add another section in any position (Fig. 2.31a).

(2) We may draw eyelid figure in any position (Fig. 2.24).

(3) We may draw a figure containing segments (Fig. 2.31b).

(4) We may draw a picture of a surface from which a disk is removed. (See Fig. 2.18a.)

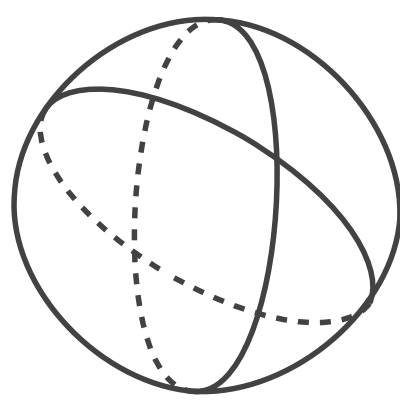

(a)

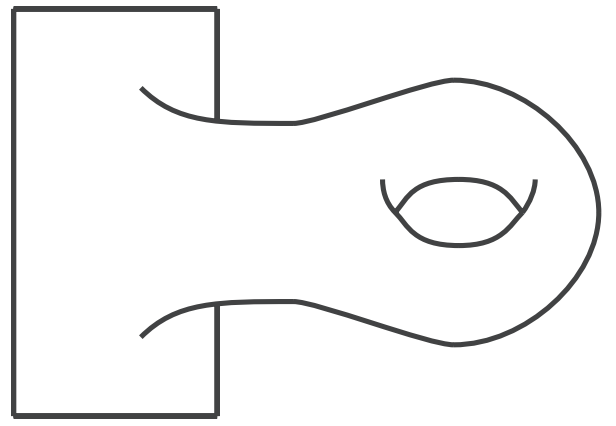

(b)

Fig. 2.31

The following shape parameters are available.

- None

Nothing is drawn.

- Oval

A full oval.

- Oval (half dotted line)

A full oval with half dotted and half solid.

- Half oval

A half oval.

- Eyelid An eyelid figure.

\subsubsection{Point element}

We are not allowed to set an isolated vertex in pipe structure. Because pipe structure must have an edge and a radius parameter. So we need another element for drawing a marked point on the drawing space as Point element. When we draw a mark on a surface, when we emphasize a singular point, we may use Point element.

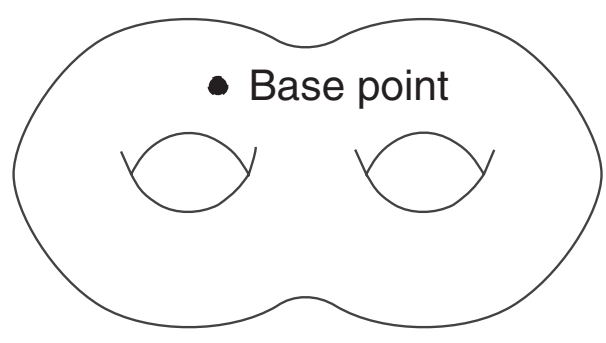

Fig. 2.32.

\subsubsection{Text}

When we want to write a text in the drawing space, we may use Text element. We can write a plane text, but we are not allowed to write a $\mathrm{T}_{\mathrm{E}} \mathrm{X}$ source. 


\section{Data Structure of a Figure}

\subsection{Bone-vertex and edge}

As mentioned in Sect. 2.4, A bone is a planer graph with straight edges. It follows that it is convenient to make the following two classes, Vertex and Edge. In this section, we see the members of these classes and how to construct pipe structure in the source.

\subsection{Class vertex}

In the class Vertex, there are following members. This class manages all of the data of vertices of a bone.

1. Data of the vertices are stocked in the linked list structure method, but other than that, we use a serial number for each vertex. The serial numbers needn't be successive.

2. This class has the coordinate of the vertex in the drawing space.

3. This class has the serial numbers of edges that meet at the vertex. For each vertex, the degree must be 3 or less, so this variable is a sequence of the length 3 .

4. This class has a boundary shape parameter. See Sect. 2.6.

5. This class has a boundary view parameter. See Sect. 2.6.

6. This class has a flag for the outline to be dotted.

7. This class has a ratio of fadecone boundary shape. See Sect. 2.6.

8. This class has a flag for the vertex to be highlighted.

Using mouse, users may change 2 and 8. From Property dialogue window, we may change 2, 4, 5, 6, 7, 8.

\subsection{Class edge}

In the class Edge, there are following members. This class manages all of the data of edges of a bone and gauges.

1. Data of the edges are stocked in the linked list structure method. And each edge has a serial number. Serial numbers needn't be successive.

2. Each edge has two end vertices. This class has the serial numbers of the end vertices of the edge.

3. This class has a pinching shape parameter. See Sect. 2.7.

4. This class has a section view parameter. See Sect. 2.7 .

5. This class has a variable of the length of the gauge.

6. This class has a position variable (, that is, the ratio in the edge) of the gauge. Default value is 0.5.

7. This class has the coordinates of two ends of the gauge. These values are automatically renewed from 2,5 , and 6.

8. This class has a flag for the edge to be highlighted.

Using mouse, we may change 5, 6, and 8. From Property dialogue window, we may change 3, 4, 5, 6, and 8.

\section{Algorithms}

\subsection{Validity of a bone}

To verify whether a bone is valid, we check the followings in this order for each vertex. Let a vertex be $V$, and let neighbor vertices of $V$ be $V_{i}(i=0,1,2)$.

(1) The edge $V V_{i}$ doesn't intersect other edges transversally in the interior of edges.

(2) There are no vertex on the edge $V V_{i}$.

(3) The length of $V V_{i}$ is not zero.

(4) There are no other edges such that the intersection is a segment.

\subsection{Add edge, move vertex}

Adding a new edge:

If we drag a mouse in New edge mode, then we can draw a new edge of a bone. If we release the mouse button on a vertex, then we can draw an edge coming to this vertex.

Moving a vertex:

If we set Move element mode and we start drag on a vertex, we can make the vertex move (and so the edges which come to the vertex). If we make a vertex move to another vertex, we may make the two vertices into one vertex (only if the resulting graph is valid as a bone). We are allowed to move vertives of bones, Oval elements, Point elements, and Text elements in this mode. We are also allowed to edit the position and the length of the gauge for each edge (or of an Oval element). To change the position of the gauge, we may drag the intersection of the edge and the gauge. To change the length of the gauge, we may drag an endpoint of the gauge. 


\subsection{Selecting and deleting}

By clicking a vertex or by drawing a surrounding rectangle, we can highlight a vertex. Selecting a Delete mode, we can delete the vertex. At the same time, all edges which come to the vertex are also deleted. Moreover, if there exists an isolated vertex after deleting, then it is deleted. We can delete an arbitrary vertex, but not an arbitrary edge.

\subsection{Drawing outline}

In the following figure, from the point $A$ to the point $B$, a Bezier curve is drawn as an outline.

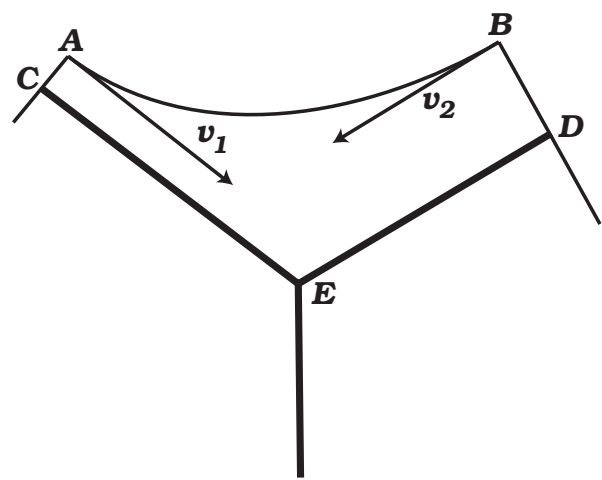

Fig. 4.1.

This outline $A B$ must be perpendicular to gauges at $A, B$. But there are no other conditions to satisfy. So there is ambiguity here. If we change the length of the tangent vectors $v_{1}, v_{2}$ at $A, B$, we have the different kind of figure. See the followings.

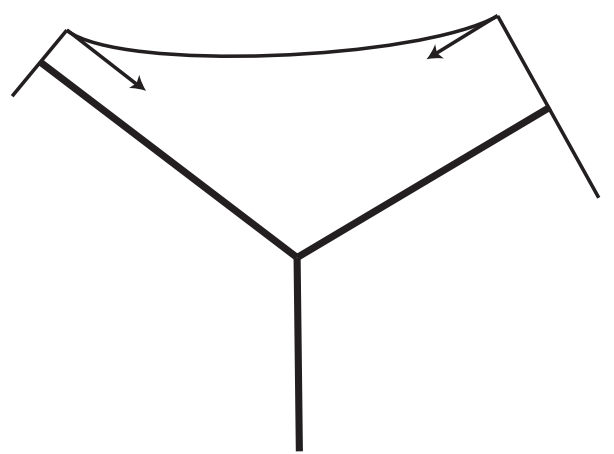

Short tangent vectors

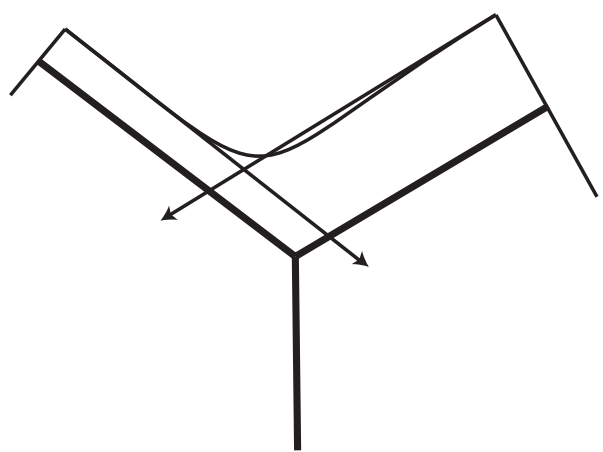

Long tangent vectors

We shall think what is the most natural length of the tangent vectors. It is clear that the length depends on the lengths of the edges, gauges, and on the angle between edges. Let the angle $\theta$ be $\angle C E D$ in Fig. 4.1. Let the tangent vector be $v_{1}, v_{2}$ at $A, B$ respectively. The direction of $v_{1}, v_{2}$ are perpendicular to $A C, B D$ respectively. The author experiment on the most natural length of $v_{1}, v_{2}$, and we get:

$$
\begin{aligned}
& \left|v_{1}\right|= \begin{cases}0.8|C E| & \text { if } \theta>\pi) \\
\max \left\{0.3,0.7-\frac{|A C|}{|C E|}\right\} \cdot|C E| & \text { (if } \theta \leq \pi)\end{cases} \\
& \left|v_{2}\right|= \begin{cases}0.8|D E| & \text { if } \theta>\pi) \\
\max \left\{0.3,0.7-\frac{|B D|}{|D E|}\right\} \cdot|D E| & (\text { if } \theta \leq \pi)\end{cases}
\end{aligned}
$$

There are no proof for this result.

\subsection{Clockwise algorithm}

As remarked in Sect. 2.2, we have to see the orientation of edges for degree 3 vertex. See Fig. 2.16. To determine the orientation, first we take the normalized vectors $\overrightarrow{A B^{\prime}}, \overrightarrow{A C^{\prime}}, \overrightarrow{A D^{\prime}}$ of $A B, A C, A D$ respectively, and calculate the signature of area of $\triangle B^{\prime} C^{\prime} D^{\prime}$. In fact, Let $\left(x_{1}, y_{1}\right),\left(x_{2}, y_{2}\right),\left(x_{3}, y_{3}\right)$ be normalized vector. That is, 


$$
\left\{\begin{array}{l}
\left(x_{1}, y_{1}\right)=\overrightarrow{A B^{\prime}}=\frac{\overrightarrow{A B}}{|A B|} \\
\left(x_{2}, y_{2}\right)=\overrightarrow{A C^{\prime}}=\frac{\overrightarrow{A C}}{|A C|} \\
\left(x_{3}, y_{3}\right)=\overrightarrow{A D^{\prime}}=\frac{\overrightarrow{A D}}{|A D|}
\end{array}\right.
$$

And the area $\triangle B^{\prime} C^{\prime} D^{\prime}$ is given by

$$
\frac{1}{2}\left(\left(x_{2}-x_{1}\right)\left(y_{3}-y_{1}\right)-\left(x_{3}-x_{1}\right)\left(y_{2}-y_{1}\right)\right)
$$

\subsection{Affine translation to draw boundaries}

To draw boundary view and boundary shape, it is good to apply affine maps.

A map $f: V \rightarrow W$ is called an affine map if

$$
f(x, y)=(a x+b y+p, c x+d y+q)
$$

for real numbers $a, b, c, d, p, q$. Here $V \cong W \cong \mathbb{R}^{2}$ and $V$ is a Euclidean 2-space and $W$ is the drawing plane. We first make data of Boundary shape and Boundary view in $V$. This example is a picture for Hyperbolic-Oval2 figure.

For each degree 1 vertex $A, a, b, c, d, p, q$ are defined as follows. Let $A D$ be a bone and Let $E F$ be the gauge. Let $B C$ be a segment such that $A$ is the midpoint of $B C$ and $\overrightarrow{B C}=\overrightarrow{E F}$.

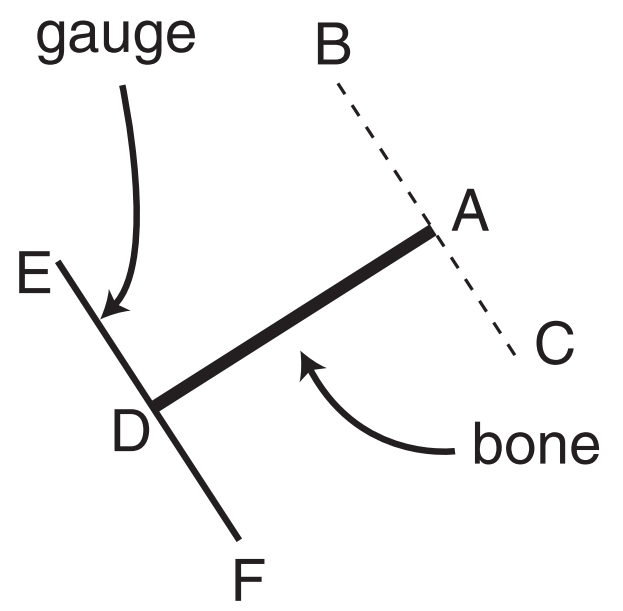

Let $(p, q)$ be the coordinate of $A$. Let $(a, c)$ be a vector $\overrightarrow{A B}$ and Let $(b, d):=(-c, a)$. It follows that $f(0,0)=A$, $f(1,0)=B$, and $f(-1,0)=C$, and $f$ is an orthnormal affine map. And we transfer the figure of the boundary. Resulting curve is as follows.

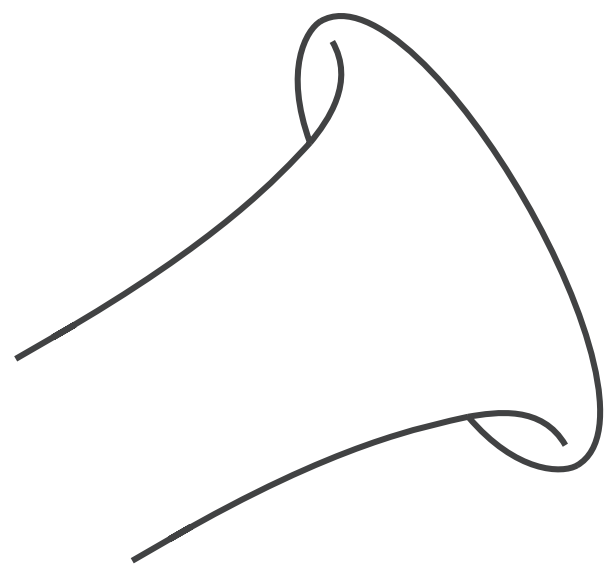




\subsection{Exporting to PostScript}

In Elephant we can save a figure into a PostScript file (***.eps). This feature allows us to export pictures which we draw with Elephant to other drawing software or to insert the figure into a $\mathrm{T}_{\mathrm{E}} \mathrm{X}$ documentations.

We use only the following three commands of PostScript.

newpath $x_{1} y_{1}$ moveto $x_{2} y_{2}$ lineto stroke : To draw a segment

newpath $x_{1} y_{1} \quad r \quad 0360$ arc stroke : To draw an oval

newpath $x_{1} y_{1}$ moveto $x_{2} y_{2} x_{3} y_{3} x_{4} y_{4}$ curveto stroke : To draw a Bezier curve.

\section{Samples}

\subsection{Two dimensional manifold in topology}
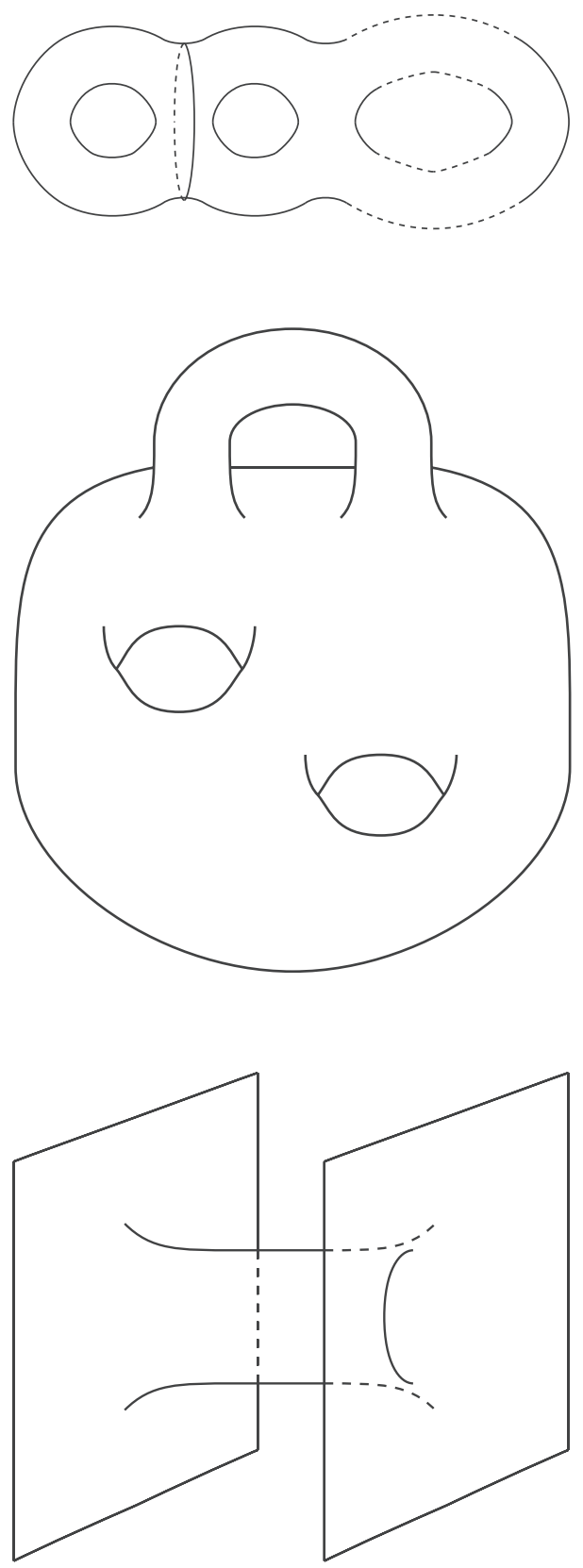


\subsection{Complex line in algebra}
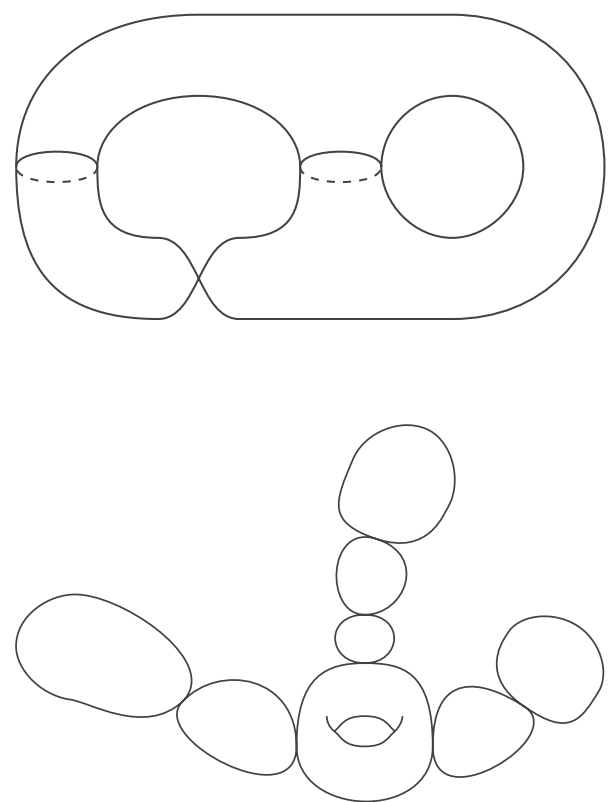

5.3 Hyperbolic surface in hyperbolic geometry

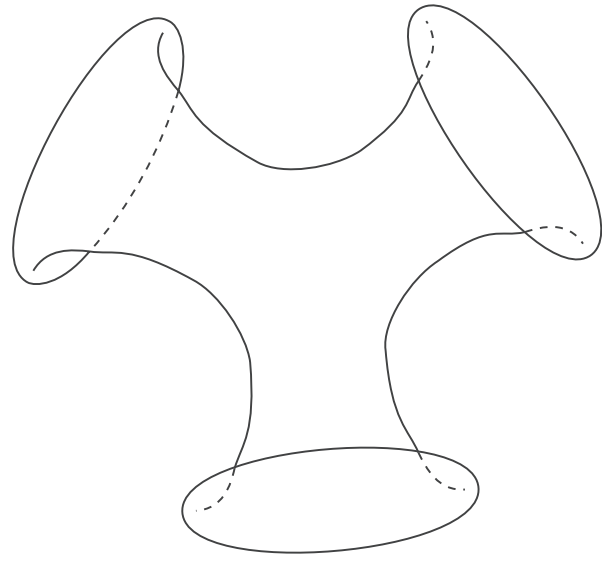

REFERENCES

[1] Adobe Illustrator is the trademark of Adobe Systems Incorporated.

[2] PostScript is a trademark of Adobe Systems Incorporated.

[3] $\mathrm{T}_{\mathrm{E}} \mathrm{X}$ is a trademark of American Mathematical Society.

[A] http://www.geocities.co.jp/CollegeLife-Labo/9021/

[Y] Yamazaki, Y., "Drawing software of Riemann surfaces and its algorithm," Master thesis of Meiji University, 2002. 